\title{
Multimodal Pain Management in the Perioperative Setting
}

\author{
Marian Sherman ${ }^{*}$, Samir Sethi ${ }^{2}$, Anna Kate Hindle ${ }^{3}$, Tamanda Chanza ${ }^{4}$ \\ ${ }^{1}$ George Washington University Medical Center, Washington DC, USA \\ ${ }^{2}$ Cardiac \& Critical Care Fellow, New York Presbyterian/Weill Cornell Medical Center, New York, NY, USA \\ ${ }^{3}$ School of Medicine and Health Sciences, George Washington University, Washington DC, USA \\ ${ }^{4}$ Anesthesia Resident at University of Maryland SOM, Baltimore, MD, USA \\ Email: ^msherman@mfa.gwu.edu
}

How to cite this paper: Sherman, M., Sethi, S., Hindle, A.K. and Chanza, T. (2020) Multimodal Pain Management in the Perioperative Setting. Open Journal of Anesthesiology, 10, 47-71.

https://doi.org/10.4236/ojanes.2020.102005

Received: December 30, 2019

Accepted: February 10, 2020

Published: February 13, 2020

Copyright (C) 2020 by author(s) and Scientific Research Publishing Inc. This work is licensed under the Creative Commons Attribution International License (CC BY 4.0).

http://creativecommons.org/licenses/by/4.0/

\begin{abstract}
In the context of the current U.S. opioid crisis, there is renewed interest and heightened relevance in the practice of multimodal analgesia for postoperative surgical patients. The goal of this review article is to present an accounting of the available opioid and non-opioid pharmacologic agents, regional analgesia techniques, and complementary modalities that may be considered for optimal pain relief in the perioperative setting. In adhering to the practice of multimodal pain therapy, clinicians improve the quality of pain control and reduce reliance on opioid use. There is strong evidence to support the benefits of multimodal analgesia.
\end{abstract}

\section{Keywords}

Multimodal Pain Management, Postoperative, Perioperative Setting, Adjuvant Analgesic Agents

\section{Introduction}

\section{Multimodal Pain Management}

This review article addresses the comprehensive approach to acute pain management in the perioperative setting. In particular, the discussion highlights multi-modal treatment strategies relevant to managing postoperative pain in patients undergoing common outpatient and inpatient surgeries. Traditional opioid therapy is reviewed, and many adjuvant analgesic agents are discussed individually. Procedural interventions such as neuraxial modalities and peripheral nerve blocks are addressed as important considerations for comprehensive perioperative pain management. Additionally, complementary modalities 
such as Virtual Reality, Music Therapy and Acupuncture are presented. Finally, acute postoperative pain management is considered in the context of the patient with a history of chronic pain.

\section{Discussion}

\section{Understanding Pain}

The International Association for the Study of Pain introduced in 1979 the most commonly used definition of pain. The IASP described pain as an unpleasant sensory and emotional experience associated with actual or potential tissue damage [1]. The IASP definition encompasses pain broadly, and it includes pain derived by nociceptive insult as well as pain that is neuropathic in origin. The definition suggests the complex nature of pain which is appropriately understood to encompass dimensions beyond merely the physiologic. In fact, to properly treat pain, the clinician must address the impact of the pain experience on a patient's quality of life. Indeed, poorly controlled pain has been shown to cause detrimental effects in all aspects of quality of life. This negative impact has been found to span every age and every type and source of pain in which it has been studied [2].

\section{The Impact of Pain}

There are approximately 50 million ambulatory surgeries and non-surgical procedures performed annually in the United States and approximately 20 million annual inpatient surgeries [3]. Nearly all patients who undergo surgery or non-surgical procedures experience pain. Although clinicians anticipate postoperative pain and possess, more than ever before, a deeper understanding of pathophysiology and pharmacology of nociception, clinicians continue to ineffectively manage postoperative pain. Approximately $50 \%-80 \%$ of patients report inadequate pain control following surgery [4]. Of these patients, an estimated $75 \%$ experience moderate or severe pain in the immediate postoperative period. Nearly $60 \%$ of patients report post-operative pain as their greatest concern prior to surgery [5].

The significance of poorly controlled pain is multifaceted. Importantly, poorly relieved pain results in patient discomfort and suffering, both of which contribute to physiologic and psychological consequences. Acutely, pain and anxiety precipitate the surgical stress response and the resulting heightened activity of the neuroendocrine system may lead to adverse perioperative outcomes. Ineffectively managed post-operative pain is associated with deep vein thrombosis, pulmonary embolism, hypertension, tachycardia, coronary ischemia, myocardial infarction, pneumonia, hyperglycemia, poor wound healing, and insomnia [6]. Additionally, high post-surgical pain scores correlate with increased hospital length of stay, delayed ambulation, long-term functional impairment, and the development of chronic post-surgical pain [6] [7] [8]. Left untreated, acute pain can lead to long-term emotional and psychological distress and has the potential to develop into chronic pain which is far more challenging to manage. Though 
the development of Chronic Post Surgical Pain (CPSP) is complex and not well understood, it is accepted that the process involves biological, psychological and socio-environmental factors. In a meta-analysis of prospective studies that evaluated pain at 3 months and 6 months post-thoracotomy, 57\% and $47 \%$ of patients, respectively, reported chronic pain [9]. Bayman et al. found that the strongest predictor of chronic pain at 6 months was intensity of acute postoperative pain [9]. Finally, a Cochrane systematic review and meta-analysis revealed that regional anesthesia reduced pain scores in patients who underwent thoracotomy and in patients who underwent mastectomy, and in both groups of patients, the development of post-surgical pain was prevented in one in four to five patients treated [10]. It has thus been established that the vast majority of the more than 50 million patients who undergo surgery annually, report moderate to severe postoperative pain.

\section{Concept of Multimodal Analgesia}

Clearly, the medical community must turn its attention to strategies that optimize the perioperative patient experience by developing a more successful approach to pain management in the perioperative period. The concept of balanced or multimodal analgesia was first introduced decades ago, and the concept rejects the notion that monotherapy (the use of a single drug alone), is an adequate approach to acute pain management and instead claims that the combination of several analgesics offers superior pain control [11]. Multimodal analgesia integrates the use of several analgesic medications, each of which targets a different pain-related receptor, and thereby exhibits its pain reducing effect by way of a different mechanism of action. When two or more analgesic medications are combined for pain relief, it allows for lower doses of each drug to be administered and thus minimizes the risk of adverse drug effects [12].

The application of multimodal pain management to current perioperative clinical practice, however, has been slow and inadequate. One departure from focus on its implementation and practice resulted from national attention paid to a Joint Commission for Accreditation of Healthcare Organizations (JCAHO) proposal in July 2000 which called for a new standard for pain management.

\section{Pain as a 5 th Vital Sign}

JCAHO aimed to address the widespread problem of underassessment and undertreatment of pain, and introduced standards for healthcare organizations to improve care for patients with non-cancer pain [13]. JCAHO proclaimed that poor pain control is unethical, clinically unsound, and economically wasteful, and by declaring pain as the $5^{\text {th }}$ vital sign, JCAHO mandated that hospitals respond actively to pain management.

In response to the new standards, the medical community relied heavily on readily available opioids to reduce post-operative pain. While the misguided use of escalating monotherapy (the use of opioids alone to treat postoperative pain) addressed immediately the concern for poorly controlled acute and chronic pain in the US healthcare system, the over-reliance on opioids ultimately contributed 
in part to the development of an epidemic of opioid abuse, diversion and addiction in the United States [14].

\section{Opioid Crisis in the United States}

A brief look at some startling facts about opioid use in the United States in the past two decades should compel healthcare providers, the authors of opioid prescriptions, to consider carefully the necessity of prescription opioids, patient selection when prescribing opioids, and finally, the duration of treatment if opioids are deemed beneficial and necessary.

- Sales of opioid analgesics quadrupled between 1999 and 2010. This represents an increase from $96 \mathrm{mg}$ morphine equivalents per person in the United States in 1997 to $710 \mathrm{mg}$ per person in 2010. This is equivalent to $7.1 \mathrm{~kg}$ of opioid medication per 10,000 persons, or enough to supply every adult American with $5 \mathrm{mg}$ of hydrocodone every 6 hours for 45 days [14]. A 2011 IMS report quantifying number of patients receiving selected pharmacologic therapies shows Narcotic Analgesics as $4^{\text {th }}$ commonest therapy (15.6 million), behind Antihypertensives (42.4 million), Lipid Regulators (19.8 million), and Antidepressants (18.5 million) [14]. Overdose deaths related to prescription opioids has also quadrupled.

- An estimated 25 million people initiated nonmedical use of pain relievers between 2002 and 2011 [15].

- The number of deaths per year attributed to prescription opioid medications reached 16,651 in 2010 [16]. In the year 2015, that number remained steady and was reported by the CDC as more than 15,000. Recent cumulative data suggests that the number of people who have died as a result of opioid overdose between 1999 and 2015 is 183,000 (Prescription opioid overdose data. 2017).

It is clear that now, more than ever, healthcare providers must responsibly subscribe to the practice of multimodal analgesia. In fact, a major goal of multimodal analgesia is the development of a satisfactory and safe regimen for pain control that is opioid-sparing in nature. Randomized trials have demonstrated that multimodal analgesia, using simultaneously more than one type of pain medication, or using one or more medications administered by different routes (systemically and neuraxially for example), offers superior pain relief and decreased opioid consumption, when compared to use of a single medication, by a single route [17] [18] [19] [20].

Some clinicians have even proposed the benefits of opioid-free analgesia (OFA) highlighting avoidance of common opioid side effects including nausea, vomiting, delayed gut motility, constipation, ileus, delirium, sedation and respiratory depression [21], and avoidance of acute opioid tolerance and hyperalgesia [22] [23] [24]. Furthermore, there is growing interest in the association between opioid administration and cancer recurrence in the surgical oncology population, specifically in patients with breast and prostate cancer [25] [26]. It is suggested that opioids suppress cellular and humoral-mediated immunity by re- 
ducing natural killer cell cytotoxicity, and may thereby increase the likelihood of cancer recurrence.

\section{Imperative Timing for the Implementation of Multimodal Analgesia}

Continuing to prescribe opioid monotherapy to control postoperative pain not only suggests denial of a health epidemic in our country, but it also reflects an incomplete understanding of the physiology of pain. In order to implement appropriate pharmacologic choices and procedural interventions for pain control, the clinician must appreciate the complexity of pain.

\section{Physiology of Pain}

Pain is a complicated process that until recently was poorly understood. We now know that noxious stimuli are first sensed by special receptors known as nociceptors. These cells transform these stimuli into electrical signals which are conducted to the spinal cord via two specialized fibers, known as a-delta and c fibers [27]. A-delta fibers are lightly myelinated and small in diameter and are responsible for transmission of acute pain. C fibers, however, which are believed to activate a slow, burning pain, are unmyelinated and therefore have slow conduction [28]. Both of these fibers synapse in the dorsal horn of the spinal cord where complex interactions occur between afferent neurons, inhibitory neurotransmitters like GABA and Glycine, and descending pathways from the cortex. From the dorsal horn pain signals are carried via two pathways to the cortex, the spinothalamic tract and the spinoreticular tract. These tracts each travel through many areas of the brain, including the brainstem reticular formation and the periaqueductal grey matter before eventually reaching the cortex. Activation of nociceptors is mediated by chemicals that are released in response to cellular or tissue damage. For example, injured cells release leukotrienes and potassium, platelets release serotonin, and vascular injury results in the release of bradykinin from plasma [29]. These nociceptive signals release neurotransmitters and substances which amplify the responses from the dorsal horn neurons, with resultant further input into the dorsal horn. Such substances include substance P, calcitonin gene-related peptide, and the excitatory amino acids, glutamate and aspartate [30].

In certain patients, these nociceptive pathways become enhanced resulting in increased excitability in response to the same stimulus. This hypersensitization is termed central sensitization and plays a key role in both acute and chronic pain [31]. Central sensitization can occur for a variety of reasons, ranging from preoperative pain to intraoperative tissue injury and postoperative inflammatory processes that can occur immediately after surgery up to several weeks later [32] [33]. Central sensitization provides the neurobiological basis for much of the pain suffered by patients long after normal tissue healing has occurred and is a cause of increased pain sensitivity described as hyperalgesia [33].

\section{Evaluating Pain}

Perioperative pain management is a dynamic process. Clinicians must continuously evaluate and adjust the pain management plan on the basis of adequacy 
of pain relief and the presence of adverse symptoms. A number of pain assessment tools have been validated for accuracy in detecting the presence of and quantifying the severity of pain, and have been tested for intrapatient and inter-rater reliability. Perhaps most commonly used because of its simplicity to administer, the Numeric Rating Scale (NRS) is a unidimensional measure of pain intensity used to evaluate pain in cognitively-intact, adult patients [34] [35]. The 11 point NRS is actually a segmented numeric version of the Visual Analog Scale (VAS) in which the clinician asks the patient to select a whole number (0 10 integers) that best reflects the intensity of his/her pain. This evaluation should be assessed in the context of pain at rest and pain with movement in order to ensure that the pain management plan best facilitates return to appropriate functional status.

\section{Identifying Patients at Elevated Risk for Postoperative Pain}

It is desirable to preoperatively identify patients who may be at higher risk for developing severe postoperative pain from those who may have lower risk. Through early identification of high risk patients, clinicians are better able to determine patient-specific plans for managing postoperative pain. Several studies have explored preoperative risk factors associated with greater acute postoperative pain [36] [37] [38] [39]. Consistent and strong associations were found for increased intensity of postoperative pain and the following variables: younger age, higher level of preexisting pain, mood disturbances such as anxiety and depression, and type of surgery. The most painful operations are orthopedics with major joints surgery, thoracic surgery, and open abdominal surgery [36] [38]. Additionally, patients who undergo bilateral breast surgery consistently report higher scores on all postoperative pain measures compared to those who undergo unilateral surgery, and women receiving immediate reconstruction (tissue expanders and implants) report more physical discomfort and dysfunction than those women who undergo delayed reconstruction (DIEP, PTRAM, and SIEA flaps) [37]. Gender was not consistently found to be a significant predictor as traditionally believed. It is evident that an increased awareness of the importance of the role that psychological factors play in the pain experience, and early identification of predictors in patients at risk for postoperative pain, will allow for more targeted intervention and more successful pain management.

\section{Multidisciplinary Communication and Patient Expectations}

The anesthesiologist's responsibility is to plan preoperatively for comprehensive perioperative patient care that integrates best pharmacologic practice and relevant procedural interventions with the goal of controlling and minimizing pain after surgery. It is unrealistic to anticipate that patients will be pain free after surgery, thus it is imperative that the perioperative team (anesthesiologist, surgeon, advanced practitioner, and nurse) discuss and clarify with the patient reasonable expectations for pain control and postoperative function. A multimodal approach which broadly considers pharmacologic agents (such as acetaminophen, nonsteroidal anti-inflammatory drugs (NSAIDs), opioids, adjuvant 
medications such as anticonvulsants, NMDA antagonists, alpha 2 agonists, local anesthetics, and others) as well as procedural interventions (such as spinals, epidurals, paravertebrals, and peripheral nerve blocks and field blocks) is recommended. Additionally, there may also be a role for complementary strategies such as transcutaneous nerve stimulation (TENS), acupuncture, music therapy, and virtual reality. Individual components of the multimodal approach to postoperative pain control will be addressed in further detail.

\section{Opioids}

Opioids represent a class of analgesics that provide powerful dose-dependent pain relief for patients suffering moderate to severe pain [40]. The class includes many compounds with variable pharmacokinetics and pharmacodynamics, no hepato-renal toxic effects, no ceiling effect for achievable pain relief, and are approved for many routes of administration, including oral, nasal, parenteral, transdermal and neuraxial administration [41]. While optimal postoperative pain management is achieved through the practice of multimodal analgesia, systemic opioids remain a cornerstone in the management of postsurgical pain.

Opioid compounds interact with four opioid receptor subtypes, designated as mu, kappa, delta and sigma [42]. A newer receptor classification utilizes the labels OPR1, OPR2, and OPR3, corresponding to $\mathrm{mu}$, kappa, delta receptors, respectively. The properties of a specific opioid depend upon which receptor it binds and the affinity with which it binds the receptor. Further, the efficacy of a full agonist (opioid) such as fentanyl is greater than that of a mixed agonist-antagonist such as nalbuphine. While opioids exert their greatest effect in the central nervous system, a lesser number of opioid receptors have been identified in peripheral tissues (i.e. GI tract, kidney).

\section{Parenteral Opioid Therapy}

In the immediate postoperative period, parenteral opioids are preferred over oral opioids because the intravenous route offers greater bioavailability and more immediate onset of analgesia. Furthermore, patients may not be able to tolerate oral medication due to the surgery performed or due to postoperative nausea and vomiting. Patient controlled analgesia (PCA) is often prescribed for patients who undergo surgeries associated with significant postoperative pain. When compared to conventional intravenous and/or intramuscular nurse-administered opioid therapy, the use of intravenous PCA affords improved analgesia, higher patient satisfaction and decreased pulmonary complications [43]. Studies that compared PCA with and without a background infusion (also known as a basal rate), have not shown improved pain relief or sleep, yet these studies have reported a significant increase in the incidence of side effects, including respiratory depression [44]. Therefore, in nearly all cases, it is not recommended to use background infusions when administering PCA analgesia. Finally, calculation of parenteral opioid requirement during the early postoperative interval can be used to guide conversion to an appropriate, equivalent oral opioid regimen.

World Health Organization Analgesic Ladder 
As soon as patients can tolerate a clear liquid diet, the analgesic regimen should be converted to oral pain medications. In 2010, a study re-examined the World Health Organization (WHO) analgesic ladder to determine if the long-used approach to pain management in patients with cancer remained relevant in current practice [44]. It was determined that several basic principles indeed remained applicable to clinical practice today, and furthermore, the basic principles were relevant to the management of patients with acute pain and chronic pain, unrelated to cancer pain [44]. The basic tenets of the WHO analgesic ladder are listed below:

1) Oral administration of analgesics is preferred whenever possible (over intravenous and intramuscular)

2) Analgesics should be given at regular intervals (when continuous pain is anticipated, "around the clock" dosing is more efficacious than "as needed")

3) Analgesics should be prescribed according to pain intensity as evaluated by a scale of intensity of pain (the determination is made after conducting a clinical examination and assessment of pain, and this tenet acknowledges patient autonomy and patient's report of pain)

4) Dosing of pain medication should be adapted to the individual (there is no standardized dosage; all patients respond uniquely)

5) Analgesics should be prescribed with a constant concern for detail (adequacy of pain control should be reevaluated regularly and adjusted as needed; collaborate with the patient)

\section{Chronic Opioid Exposure and Opioid Induced Hyperalgesia}

Given the prevalence of both licit and illicit opioid use, there are a growing number of chronic opioid-consuming patients who present for surgery. This population of patients presents a unique challenge to the perioperative team. $\mathrm{Pa}$ tients who use opioids chronically are expected to experience increased postoperative pain and consume greater opioid doses, and these patients require prolonged use of healthcare resources for managing their pain [45].

Chronic exposure to opioids commonly results in an increased opioid requirement over time to maintain the desired analgesic effect. The need for escalating the dose is often attributed to the development of tolerance. More recent evidence points to an alternative neuropharmacological phenomenon that may explain the need to escalate the opioid dose over time, namely, opioid-induced hyperalgesia $(\mathrm{OIH})$. The phenomenon offers that the administration of opioids may paradoxically induce an increased sensitivity to pain, also known as hyperalgesia [22]. OIH can be viewed as a state of facilitated nociceptive signaling. Further complicating matters, the phenomena of tolerance and hyperalgesia can coexist [45] [46]. It is important to note that while OIH is commonly discussed in the context of patients with chronic pain, OIH has been observed to develop acutely, namely during the intraoperative course when short-acting opioids such as remifentanil are infused over several hours [23]. These complex pharmacological challenges should serve as reminders to clinicians that, just as we refer to 
our cardiology colleagues for expertise in the management of patients with complex cardiac disease, patients with chronic pain who present for surgery may benefit from consultation with Acute Pain specialists. Early involvement allows for preoperative interventions (both pharmacologic and procedural) that may benefit short and long term outcomes. Many hospitals have Acute Pain Management services that can provide expert review of complex patients and recommendations for successful management of these patients.

\section{Multimodal Adjuvant Analgesics}

\section{Acetaminophen}

For many decades, acetaminophen has been a common agent used in the multimodal approach to perioperative pain management. While it mechanism of action is still not completely understood, acetaminophen is thought to provide analgesia by centrally inhibiting the effects of certain prostaglandins. Some theorize that it may also inhibit the formation of inflammatory markers, including histamine, leukotrienes, and cytokines involved in the pain pathways. Others believe that it acts on the serotonin pathways which affect spinal nociception [47]. Regardless of its mechanism, multiple studies have shown improved analgesia, decreased opiate consumption, and reduced postoperative nausea vomiting when used perioperatively.

Acetaminophen, available in both oral and intravenous formulations, can be used independently for moderate postoperative pain relief or in conjunction with opiates for severe pain relief. Oral acetaminophen when dosed up to 1 gram preoperatively, has been shown to be a safe and effective method of reducing postoperative pain in a wide variety of cases, including laparoscopic/open gynecologic cases, short general surgery cases, same day surgeries, and ophthalmology cases [48].

IV acetaminophen has been studied in major surgical cases and in the intensive care unit and has shown statistically significant reductions in acute postoperative pain. In one recent meta-analysis of 14 randomly controlled trials in cluding more than 1400 patients receiving IV acetaminophen, 12/14 studies showed improved analgesia and 10/14 studies showed decreased opiate consumption [49]. Most major studies have shown benefits at a dosing of 1 gram every 6 to 8 hours, with a max total of 3 - 4 grams per day.

Many providers and hospitals do not have access to IV acetaminophen intraoperatively due to its higher cost when compared to oral acetaminophen. While studies regarding differences in outcomes between patients receiving oral versus IV acetaminophen are currently limited and of mixed opinion, there is still evidence to support its use. Compared to oral formulations, IV acetaminophen has a much higher bioavailability and has been shown to have a significantly faster peak plasma level. IV formulations have also been shown to have reduced toxic metabolites when compared to its oral counterpart [47]. While acetaminophen has an excellent safety profile, a reduced dose should be used in patients with 
mild/moderate hepatic insufficiency, alcoholism, and in low-weight adults and adolescents. Acetaminophen should be avoided in patients with severe liver disease [50].

\section{Nonsteroidal Anti-Inflammatory Agents}

Nonsteroidal anti-inflammatory agents or NSAIDS are an effective non opiate analgesic that can be used preoperatively, intraoperatively, or postoperatively to manage postoperative pain. NSAIDS act by inhibiting the cyclooxygenase (COX) enzyme which blocks the inflammatory response by preventing the formation of prostaglandins. NSAIDS can be categorized by their selectivity of inhibition of the two COX enzymes, COX-1 and COX-2. The COX-1 enzyme has been shown to be active in most tissues, especially in platelets, kidneys, and the stomach. The COX-2 enzyme appears to be found in lower concentrations peripherally and in higher concentrations in the brain, kidneys, and bones [51].

Non-selective NSAIDS, which block both enzymes, include aspirin, diclofenac, ibuprofen, ketorolac, naproxen and many others. The side effects of nonselective NSAIDS stem mainly from its COX-1 inhibition which leads to decreased thromboxane production resulting in a significant decrease in platelet aggregation. For this reason, nonselective NSAIDS should be avoided in certain surgical cases and in certain patients. Non-selective NSAIDS also carry a risk of damage to the gastric linings, renal vasoconstriction, and acute renal failure [52]. One of the most commonly used non-selective NSAIDs is ketorolac, which is available in both intravenous and oral formulations. In multiple randomized controlled trials, ketorolac given as a single intraoperative dose of 15 - $60 \mathrm{mg}$ has shown significant reductions in postoperative pain and decreased postoperative opiate consumption [53]. This effectiveness has been demonstrated in a wide variety of cases, including cesarean sections and general surgery cases, and can be continued postoperatively as well.

\section{Selective COX-2 inhibitors}

Selective COX-2 inhibitors, designed to minimize some of the harmful effects described above, include drugs such as celecoxib, rofecoxib, and meloxicam. In multiple animal studies as well as large studies focused on arthritis patients in the nonoperative setting, selective COX-2 inhibitors have shown decreased platelet effects and decreased gastric ulceration, highlighting the potential benefits of COX-2 inhibitors over non-selective inhibitors [54]. While there is limited data on postoperative side effects of COX-2 inhibitors, it appears to be well tolerated. It is believed that patients with GI risk factors, who would otherwise not be able to take a non-selective NSAID, may benefit from the selective COX-2 inhibitors. Some clinical trials and animal studies have also suggested that nonselective NSAIDS may have a deleterious effect on bone healing, while selective COX-2 inhibitors may not, leading to another potential benefit of the selective COX-2 inhibitors. Cardiovascular effects of COX-2 inhibitors which has become a controversial topic over the last few years remains a topic for discussion and needs to be further studied. In multiple randomly controlled, double blinded 
studies, COX-2 inhibitors have shown to be superior to placebo in reducing postoperative pain and have a similar analgesic ceiling when compared to nonselective NSAIDS [55]. The most commonly used COX-2 inhibitor studied today is celecoxib dosed at 200 or $400 \mathrm{mg}$ as a single dose preoperatively with the option of continuing postoperatively [52].

\section{Gabapentin and Pregabalin}

Gabapentin and pregabalin are alkylated-aminobutyric acid analogs that were first developed clinically as anticonvulsant agents. Both medications target and bind an auxiliary subunit of voltage-gated calcium channels and exert their effect by reducing lesion-induced hyperexcitability of posterior horn neurons, which is a proposed mechanism responsible for central sensitization [56]. The mechanism of the antihyperalgesic action may be a result of the postsynaptic binding of gabapentin to the alpha2-delta subunit of the dorsal horn neurons' voltage-dependent calcium channels, causing decreased calcium entry into nerve endings and thus decreased release of neurotransmitters, including glutamate, substance $\mathrm{P}$ and noradrenaline [56]. These anticonvulsants have been shown to enhance the analgesic effect of morphine, NSAIDS, and selective COX-2 inhibitors [40] [51] [57]; and they have been shown to produce anti-hyperalgesia [58].

Gabapentin and pregabalin have been shown to exert opioid sparing effects and reduce pain scores in a number of different surgical populations. For example, in patients undergoing coronary artery bypass graft surgery with median sternotomy as well as internal mammary artery harvesting, gabapentin administered preoperatively and continuously for two postoperative days resulted in significantly reduced post-op pain intensity for three post-operative days and significantly reduced IV tramadol rescue during that time [59]. In a meta-analysis that included six studies and more than 700 patients, the effect of gabapentin on acute postoperative pain was examined in patients undergoing total knee arthroplasty. The authors concluded that gabapentin exerts an analgesic and opioid-sparing effect in acute postoperative pain management without increasing the rate of dizziness and pruritus [60]. Similarly, in patients who underwent total hip arthroplasty, pregabalin administered preoperatively and continued twice daily for seven days following surgery, resulted in significantly improved pain scores on days 1 - 7 after hospital discharge, and the pregabalin group required less adjunctive opioid medication (Percocet) 1 week after hospital discharge [61]. Finally, when compared to a placebo group, patients who received preoperative administration of gabapentin before abdominal hysterectomy, reported lower VAS scores at 1, 4, 6, 12 and 24 hours postoperatively, consumed significantly lower rescue analgesics, and experienced significantly lower incidence of PONV [62].

Finally, it has been suggested that preoperative dosing of gabapentin or pregabalin may be particularly beneficial for patients who are opioid-tolerant and are undergoing major surgery or other surgeries associated with substantial pain [63]. Overall, these medications have been reported to be a well-tolerated, safe, 
and efficacious [56].

\section{Alpha2 Agonists (Clonidine, Dexmedetomidine, Tizanidine)}

Alpha $_{2}$ agonists have been in clinical use for decades, primarily in the treatment of hypertension. More recently however, $\mathrm{alpha}_{2}$ agonists have found wider application, particularly in the fields of anesthesia and pain management. It has been noted that these agents can enhance analgesia provided by traditional analgesics, such as opiates, and may result in opiate-sparing effects. The clinical utility of these agents is ever expanding, as they are gaining broader use in neuraxial analgesia, and new applications are continuously under [64].

Clonidine, an alpha-2 adrenergic receptor agonist, has a well-established role in acute perioperative pain management. The role of systemic and intrathecal clonidine in acute postoperative pain was studied in a meta-analysis that included nearly 1800 patients [65]. The authors reviewed randomized placebo-controlled trials that evaluated systemic $\alpha 2$ agonists administered and their effect on postoperative cumulative opioid consumption and/or pain intensity. The authors concluded that perioperative systemic $\alpha 2$ agonists decrease postoperative opioid consumption, pain intensity, and nausea. In addition, recovery times are not prolonged. Common adverse effects include bradycardia and arterial hypotension.

The addition of alpha2 agonists to local anesthetic solution for peripheral nerve block may enhance and prolong analgesia [66]. This study evaluated the effect of clonidine compared to tramadol when added to local anesthetic solution for brachial plexus blocks. Patients undergoing upper extremity orthopedic surgery received brachial plexus blocks with a local anesthetic solution to which one of three additives was randomly added: $1 \mathrm{mg} / \mathrm{kg}$ tramadol, $1 \mu \mathrm{g} / \mathrm{kg}$ clonidine, or $1.5 \mu \mathrm{g} / \mathrm{kg}$ clonidine. Clonidine in a dose of $1.5 \mu \mathrm{g} / \mathrm{kg}$ body weight provided the fastest onset of sensory block as well as motor block, and clonidine 1.5 $\mu \mathrm{g} / \mathrm{kg}$ body weight resulted in the longest duration of postoperative analgesia. In addition, patients who received clonidine (both doses) rather than tramadol, experienced significantly less PONV.

Most of the clinical experience gained in the study of intrathecal $\alpha 2$-adrenoceptor agonists has been described with clonidine. In a study by Mohamed et al, investigators studied the analgesic efficacy of intrathecally administered dexmedetomidine or dexmedetomidine combined with fentanyl in patients undergoing major abdominal cancer surgery [67]. Study patients received a spinal anesthetic consisting of hyperbaric bupivacaine $0.5 \%$ with saline, hyperbaric bupivacaine $0.5 \%$ with dexmedetomidine, or hyperbaric bupivacaine $0.5 \%$ with dexmedetomidine and fentanyl. It was found that dexmedetomidine $5 \mu \mathrm{g}$ given intrathecally, compared to placebo, improves the quality and the duration of postoperative analgesia and also provides an analgesic sparing effect in patients undergoing major abdominal cancer surgery. Furthermore, the addition of intrathecal fentanyl $25 \mu \mathrm{g}$ revealed no valuable clinical effect.

Tizanidine, a centrally acting $\alpha 2$-agonist with muscle relaxant properties, was 
studied to determine the efficacy of tizanidine compared to placebo in patients undergoing inguinal herniorrhaphy. Tizanidine $4 \mathrm{mg}$ was administered orally 1 $\mathrm{h}$ before surgery and then twice daily during the first postoperative week. Patients who received tizanidine reported significantly lower NRS pain scores than those in the placebo group at 6,12 and $24 \mathrm{~h}$ postoperatively, both at rest and during movement, and on postoperative days 1, 2, 3 and 4. Tizanidine decreased postoperative pain, decreased analgesic consumption and improved return to normal activity and quality of life [68].

\section{Ketamine}

Named after its chemical structure of ketones and amines, ketamine has become one of the more popular non-opiate intraoperative pharmacologic therapies in recent years. Its analgesic effect has been attributed to its strong NMDA receptor antagonist properties. While not completely understood, the NMDA receptor has been thought to play a key role in nociceptive transmission, especially, central sensitization. The NMDA receptor is involved in a complicated process involving amplification of peripheral pain signals through the spinal cord pathways. It is this blunting of central sensitization that is hypothesized to explain ketamine's role in the prevention of postoperative and chronic pain. In addition, ketamine blocks NMDA receptors centrally, which have been shown to decrease cortical pain receptor activation on functional MRI [69].

Ketamine infusions and low dose boluses have been studied in several types of procedures including abdominal, rectal, laparoscopic, orthopedic, and spinal surgeries. Most studies have demonstrated postoperative analgesic relief with decreased postoperative opiate consumption [70]. A large meta-analysis, including 91 randomly controlled trials and more than 4000 patients, showed that perioperative IV ketamine can reduce PACU pain scores and opiate consumption and lengthen the interval of time to pain medicine request. It was found that ketamine was most effective in thoracic, upper abdominal, and major orthopedic cases [71]. Another meta-analysis of 14 randomly controlled trials including over 600 patients found that in spine surgery, the addition of perioperative supplemental ketamine reduced postoperative opiate consumption at 6,12 , and 24 hours with no statistical difference in adverse effects [72]. Of note, although studies have shown ketamine is associated with lower pain scores in the pediatric population, there fails to be any significant evidence that this reduces opiate consumption postoperatively [73].

Ketamine has also been shown to be an important agent in treating postoperative pain in opioid-tolerant patients. In one randomly controlled trial of $300 \mathrm{pa}-$ tients with chronic back pain undergoing elective lumbar surgery, patients who received ketamine intraoperatively were shown to have a $24 \%$ reduction of intraoperative opiates, a $37 \%$ reduction in postoperative opiates, and a $26 \%$ reduction in pain immediately as well as at 6 weeks postoperatively [74]. In most recent studies, ketamine was administered in both small bolus doses of 0.1 to 0.5 $\mathrm{mg} / \mathrm{kg}$ doses as well as intraoperative infusions of 2 to $4 \mathrm{mcg} / \mathrm{kg} / \mathrm{min}$. 
Although considered to be a relatively safe drug, ketamine has been noted to have some adverse reactions. Minor side effects include nausea, headache, drowsiness, and dizziness. In addition, CNS effects such as hallucinations, disorientation, dissociative effects, confusion, and vivid dreams have also been reported. At anesthetic doses, ketamine has been noted to increase blood pressure and heart rate and may potentially lead to myocardial depression. Hence, caution should be taken when using ketamine in patients with depleted catecholamines, even at sub-anesthetic doses [75]. Ketamine has also been shown to increase cerebral blood flow and cerebral metabolism. While recent evidence shows minimal increases in ICP in mechanically ventilated patients, it should still be used with caution among neurosurgical patients until further studied. [75]. Of note, hepatotoxicity has also been documented, though more commonly with doses greater than $1 \mathrm{mg} / \mathrm{kg}$ and multi day continuous infusions.

A few of ketamine's unique properties include its maintenance of respiration as well as its bronchodilator activity. This can prove helpful in cases where both analgesia and maintenance of spontaneous ventilation are necessary. It is important to note, however, ketamine does increase both salivary and tracheal secretions.

\section{Lidocaine}

Although the exact mechanism of lidocaine for intraoperative analgesia remains unclear, multiple trials and meta-analysis have shown evidence that in certain cases intravenous lidocaine can reduce postoperative pain and opiate consumption. Lidocaine is a sodium channel blocker, and it is hypothesized that its analgesic effects are related to reductions in inflammatory signaling, blocking of central neuronal excitation, NMDA receptor activity, and decreased substance P. Remarkably, studies have shown the clinical effect of lidocaine is significantly longer than the half-life of lidocaine itself, 8.5 hours vs 5.5 hours [76].

In one meta-analysis of 16 randomly controlled trials and over 700 patients, infusion of IV lidocaine in open and laparoscopic abdominal surgery showed a clinically significant reduction in pain scores for up to 48 hours postoperatively. In addition, there was earlier return of bowel function, reduction in postoperative nausea/vomiting, and shorter duration of hospital stay among lidocaine treated patients [77]. Multiple studies have shown similar results including patients undergoing open radical prostatectomies, spine surgery, thoracic surgery, as well as ambulatory surgeries [76]. In studies that showed statistically significant effective analgesia postoperatively, lidocaine infusions were run at 1.5 to 3 $\mathrm{mg} / \mathrm{kg} /$ hour following bolus dosing of up to $1.5 \mathrm{mg} / \mathrm{kg}$. Of note, there is also evidence showing that receiving lidocaine has no statistical difference in postoperative pain control. [77]n occur, especially with prolonged infusions. Symptoms of toxicity can include neurologic disturbances such as dizziness, visual changes, and lightheadedness. Severe instances of lidocaine toxicity may also manifest in convulsions and cardiac arrhythmias. While there is no strong evidence regarding the continuation of lidocaine infusions postoperatively, many practitioners report no significant side effects for up to 48 hours [77]. 


\section{Magnesium Sulfate}

Magnesium has been reported to produce important analgesic effects. While the precise mechanism of action is unclear, it is hypothesized that magnesium could modulate postoperative pain by preventing nociception associated with central sensitization via blockade of NMDA receptor calcium ionophore [78]. While magnesium indeed possesses anti-nociceptive activity as an NMDA antagonist, it has not been consistently shown to offer benefit in the perioperative setting for acute pain management. In one study of 24 patients who underwent thoracotomy, patients who received postoperative, intravenous $\mathrm{MgSO} 4$ consumed significantly less opioids [79]. In another small study of patients undergoing major lumbar orthopedic surgery, when compared to controls who received traditional opioid-based analgesics, patients who received preoperative, intravenous magnesium demonstrated decreased opioid consumption, lower pain scores, higher quality sleep on first night after surgery, and overall higher global satisfaction scores [80]. In a systematic review of fourteen randomized trials that evaluated the role of magnesium as an adjuvant to postoperative analgesia, the authors concluded that there was a lack of convincing evidence that perioperative magnesium may have favorable effects on postoperative pain intensity and analgesic requirements [81]. They did determine, however, that perioperative magnesium supplementation prevents postoperative hypomagnesemia and decreases the incidence of postoperative shivering. In contrast, a 2012 meta-analysis that reviewed 25 trials that examined perioperative magnesium administration and postoperative pain, the authors concluded that perioperative intravenous magnesium reduces opioid consumption, and to a lesser extent, pain scores, in the first $24 \mathrm{~h}$ postoperatively, without any reported serious adverse effects [82]. Given that there is such inconsistency in conclusions regarding the use of magnesium as an adjuvant agent in multimodal analgesia, it seems worthwhile to further study the role of magnesium as a supplement to postoperative analgesia, since this relatively harmless molecule is inexpensive, and the biological basis for its potential antinociceptive effect is promising [81].

\section{Procedural Interventions}

\section{Neuraxial Techniques}

Neuraxial techniques can be a valuable component of multimodal pain management. Epidural and spinal techniques may be used as an intraoperative anesthetic and may also be an important component of multimodal pain management. In this section, there will be a focus on neuraxial techniques as it pertains to multimodal pain management.

Epidural techniques may be used to improve pain control with minimal systemic opioids. An epidural infusion may be a local anesthetic alone or have other medications added for improved pain control. An opioid may be added to an epidural but it will be at significantly lower doses than if given systemically. Patients receiving epidurals for colorectal surgeries have been found to have 
quicker return of bowel function and epidurals are encouraged in many Enhanced Recovery After Surgery (ERAS) protocols [83]. Epidurals may be placed in the thoracic or lumbar area depending on the location of the painful sensation. In addition to a more rapid return of bowel function, other benefits of epidural placement may include improved pain control with minimized systemic opioid use [84]. Risks of epidural placement include but are not limited to inadvertent dural puncture, postdural puncture headache, hypotension, and inadequate pain control [83] [85]. Intrathecal morphine is also used for improved pain control after certain orthopedic procedures such as total joint replacements [86].

\section{Regional Anesthesia:}

Regional anesthesia techniques are also a valuable component of intraoperative pain management. Regional blocks may either be a single injection or may involve the placement of a catheter which will allow for a continuous dose of medication to be administered to a nerve bundle or plexus.

\section{Transversus Abdominis Plane Blocks (TAP)}

Transversus Abdominis Plane (TAP) blocks are the injection of local anesthetic between the internal and external oblique muscles to provide analgesia to the abdominal wall. TAP blocks have been also been found to decrease opioid consumption. Risks of the procedure include bowel injury but this is rare; ultrasound techniques allow for visualization of the muscular planes. TAP blocks may be useful for any open abdominal procedure and are also a valuable component of ERAS protocols [87].

\section{Paravertebral Blocks $(P V B)$}

Paravertebral blocks (PVB) may be a useful component for pain control for any procedure of the thoracic or abdominal area. A PVB is an injection of local anesthetic into the paravertebral space which allows for analgesia for thoracic or abdominal procedures [88]. Some studies have shown equivalent pain relief when the technique is compared to epidural placement. In some studies, placement of a PVB has been shown to decrease the incidence of chronic pain in patients undergoing breast surgery [89].

\section{Regional Blocks for Extremity Surgery}

There are several regional techniques that are well suited to orthopedic procedures. Certain nerve plexuses or bundles may be identified and anesthetized with perineural injection of local anesthetic. A brachial plexus block (interscalene, supraclavicular, infraclavicular or axillary approach) may be effective analgesia for an upper extremity injury. A sciatic or femoral block may allow for improved pain control in the lower extremity. Femoral nerve block has been shown to improve pain control when placed in patients undergoing total knee arthroplasty [90]. In addition, peripheral nerve blocks have been found to be helpful in reducing the incidence of phantom limb pain [91].

\section{Complementary Modalities}

\section{Virtual Reality}


Virtual reality (VR) is a technologically advanced system that allows users to be transported into a "virtual world". Multimodal (visual, auditory, tactile and olfactory) stimuli contribute to a sense of actual presence/immersion in the virtual world, thus making the VR experience distinct from passively watching television or movies [92]. Originally applied purely to the entertainment industry, VR has more recently been used to manage pain and distress associated with a wide variety of known painful medical procedures. VR has consistently been shown to decrease pain, anxiety, unpleasantness, time spent thinking about pain and perceived time spent in a medical procedure. In fact, current functional brain MRI results indicate that participants who are actively engaged in VR demonstrate inhibition of pain signaling in the critical regions of the pain matrix [93]. With technology rapidly evolving, the cost of VR decreasing, a movement to diminish reliance on traditional opioid therapy for pain control, and therefore an increased interest in multimodal pain management, including complementary non-pharmacological interventions, there is substantial potential for the use of VR in acute pain management.

\section{Transcutaneous Electrical Nerve Stimulation}

Transcutaneous electrical nerve stimulation (TENS) is a non-pharmacologic therapy in which a non-invasive, low-voltage electrical current is applied to the skin to relieve pain at the same site. TENS has been used as an adjunct pain reliever across many clinical scenarios, including cardiac surgery, cholecystectomy, cesarean delivery, and thoracic surgery. A meta-analysis of nine RCTs investigated the effect of TENS as an adjunct therapy for relieving acute post-thoracotomy pain in patients undergoing thoracic surgery [94]. The authors found that patients who had TENS therapy reported lower pain scores and used less narcotic pain medication than patients who received placebo-TENS therapy; furthermore, when used in conjunction with narcotic pain medications, patients who had TENS therapy had shorter recovery room stays and improved chest physical tolerance (better coughing attempts during chest physiotherapy) with positive effects on pulmonary ventilator function $\mathrm{FEV}_{1}$ and/or FVC). Additionally, in another study, TENS at both low and high frequencies was found to significantly reduce postoperative pain intensity in patients who underwent laparoscopic tubal ligation. Importantly, pain intensity was reduced in a clinically significant manner, from severe pain (7/10) before TENS, to moderate pain (4/10) after TENS [95].

\section{Acupuncture}

Acupuncture, a component of traditional Chinese medicine, is a well-known practice in the pain management field that involves needle penetration of the skin at certain anatomic locations identified as acupoints [96]. There have been increasing numbers of clinical trials evaluating the efficacy of acupuncture and related techniques as an adjuvant method for postoperative analgesia. In a meta-analysis incorporating data from 15 RCTs, acupuncture proved an effective adjunct for postoperative pain management as demonstrated by a significant reduction of postoperative pain scores and opioid consumption [97]. The opio- 
id-sparing effect was most marked at $72 \mathrm{~h}$ after operation where a $29 \%$ reduction of morphine consumption was demonstrated. The opioid-sparing effect at 8 and $24 \mathrm{~h}$ was $21 \%$ and $23 \%$, respectively. Furthermore, this meta-analysis showed a significant reduction in the incidence of opioid-related adverse effects, including nausea, pruritus, dizziness, sedation, and urinary retention in the acupuncture treatment group.

\section{Music Therapy}

Medical music and music therapy have been proposed as complementary modalities for improved pain control in the clinical setting. A recent meta analysis based on published RCT studies investigating the effect of music on pain between 1995 and 2014 echoed the positive findings of earlier studies [98]. Results from the meta-analysis suggest that music interventions overall have beneficial effects on pain intensity, emotional distress from pain, use of anesthetic, opioid and non-opioid agents, heart rate, systolic and diastolic blood pressure, and respiration rate. Music offers a non-invasive, complementary approach for the relief of acute, procedural, and cancer/chronic pain in the medical setting.

\section{Conclusion}

Understandably, pain is a prominent preoperative patient concern and source of anxiety. Pain in the perioperative environment is a complex phenomenon, an interaction of hyperalgesia, inflammation and, neuropathic pain, and it significantly influences patient recovery, morbidity, mortality, satisfaction, and healthcare costs. Perioperative pain is in fact pervasive. An estimated $86 \%$ of surgical patients suffer from acute postoperative pain, and the majority of patients who report pain classify their pain as moderate to extreme [5]. With advances in understanding of the pathophysiology of pain and an increased awareness of the importance of pain management, particularly in the context of a current national opioid crisis, there has never been a more compelling time to incorporate opioid-sparing, multimodal analgesia in the clinical care of peri-operative surgical patients. The optimal approach to postoperative pain management considers balanced pharmacology, regional analgesia and complementary modalities. Multimodal analgesia offers improved pain control, and importantly, the approach minimizes side effects and reduces opioid use. Further, adequate pain control in the acute setting reduces the likelihood of the development of chronic pain syndromes.

\section{Conflicts of Interest}

The authors declare no conflicts of interest regarding the publication of this paper.

\section{References}

[1] International Association for the Study of Pain (IASP) (2017) 
http://www.iasp-pain.org

[2] Duenas, M., Ojeda, B., Salazar, A., Mico, J.A. and Failde, I. (2016) A Review of Chronic Pain Impact on Patients, Their Social Environment and the Health Care System. Journal of Pain Research, 9, 457-467. https://doi.org/10.2147/JPR.S105892

[3] Cullen, K.A., Hall, M.J. and Golosinskiy, A. (2009) Ambulatory Surgery in the United States, 2006. National Health Statistics Reports, No. 11, 1-25.

[4] Apfelbaum, J.L., Chen, C., Mehta, S.S. and Gan, T.J. (2003) Postoperative Pain Experience: Results from a National Survey Suggest Postoperative Pain Continues to Be Undermanaged. Anesthesia \& Analgesia, 97, 534-540. https://doi.org/10.1213/01.ANE.0000068822.10113.9E

[5] Gan, T.J., Habib, A.S., Miller, T.E., White, W. and Apfelbaum, J.L. (2014) Incidence, Patient Satisfaction, and Perceptions of Post-Surgical Pain: Results from a US National Survey. Current Medical Research and Opinion, 30, 149-160.

https://doi.org/10.1185/03007995.2013.860019

[6] Wu, C.L. and Raja, S.N. (2011) Treatment of Acute Postoperative Pain. The Lancet, 377, 2215-2225. https://doi.org/10.1016/S0140-6736(11)60245-6

[7] Macrae, W.A. (2008) Chronic Post-Surgical Pain: 10 Years on. British Journal of Anaesthesia, 101, 77-86. https://doi.org/10.1093/bja/aen099

[8] Carr, D.B. and Goudas, L.C. (1999) Acute Pain. The Lancet, 353, 2051-2058. https://doi.org/10.1016/S0140-6736(99)03313-9

[9] Bayman, E.O. and Brennan, T.J. (2014) Incidence and Severity of Chronic Pain at 3 and 6 Months after Thoracotomy: Meta-Analysis. The Journal of Pain, 15, 887-897. https://doi.org/10.1016/j.jpain.2014.06.005

[10] Andreae, M.H. and Andreae, D.A. (2013) Regional Anaesthesia to Prevent Chronic Pain after Surgery: A Cochrane Systematic Review and Meta-Analysis. British Journal of Anaesthesia, 111, 711-720. https://doi.org/10.1093/bja/aet213

[11] Kehlet, H. (1989) Surgical Stress: The Role of Pain and Analgesia. British Journal of Anaesthesia, 63, 189-195. https://doi.org/10.1093/bja/63.2.189

[12] Fassoulaki, A., Triga, A., Melemeni, A. and Sarantopoulos, C. (2005) Multimodal Analgesia with Gabapentin and Local Anesthetics Prevents Acute and Chronic Pain after Breast Surgery for Cancer. Anesthesia \& Analgesia, 101, 1427-1432. https://doi.org/10.1213/01.ANE.0000180200.11626.8E

[13] Baker, D.W. (2017) History of the Joint Commission's Pain Standards: Lessons for Today's Prescription Opioid Epidemic. JAMA, 317, 1117-1118. https://doi.org/10.1001/jama.2017.0935

[14] Manchikanti, L., et al. (2012) Opioid Epidemic in the United States. Pain Physician, 15, ES9-E38.

[15] Gordek, H. and Folsom, R. (2012) 2011 National Survey on Drug Use and Health: Sampling Error Report. Substance Abuse and Mental Health Services Administration, Rockville.

[16] The Centers for Disease Control and Prevention. http://www.cdc.gov/nchs/data/dvs/Record_Layout_2012.pdf

[17] (2017) Prescription Opioid Overdose Data.

[18] Rawal, N., Viscusi, E., Buvanendran, A. and Kehlet, H. (2009) Multimodal minus Reuben. Anesthesiology News, 35, 239.

[19] Joshi, G.P. (2005) Multimodal Analgesia Techniques and Postoperative Rehabilitation. Anesthesiology Clinics of North America, 23, 185-202.

https://doi.org/10.1016/j.atc.2004.11.010 
[20] White, P.F., Kehlet, H., Neal, J.M., Schricker, T., Carr, D.B., Carli, F. and Fast-Track Surgery Study Group (2007) The Role of the Anesthesiologist in Fast-Track Surgery: From Multimodal Analgesia to Perioperative Medical Care. Anesthesia \& Analgesia, 104, 1380-1396. https://doi.org/10.1213/01.ane.0000263034.96885.e1

[21] American Society of Anesthesiologists Task Force on Acute Pain Management (2012) Practice Guidelines for Acute Pain Management in the Perioperative Setting: An Updated Report by the American Society of Anesthesiologists Task Force on Acute Pain Management. Anesthesiology, 116, 248-273.

[22] Hayhurst, C.J. and Durieux, M.E. (2016) Differential Opioid Tolerance and Opioid-Induced Hyperalgesia: A Clinical Reality. Anesthesiology, 124, 483. https://doi.org/10.1097/ALN.0000000000000963

[23] Kim, S.H., Stoicea, N., Soghomonyan, S. and Bergese, S.D. (2014) Intraoperative Use of Remifentanil and Opioid Induced Hyperalgesia/Acute Opioid Tolerance: Systematic Review. Frontiers in Pharmacology, 5, 108. https://doi.org/10.3389/fphar.2014.00108

[24] Guignard, B., Bossard, A.E., Coste, C., Sessler, D.I., Lebrault, C., Alfonsi, P., Chauvin, M., et al. (2000) Acute Opioid Tolerance Intraoperative Remifentanil Increases Postoperative Pain and Morphine Requirement. The Journal of the American Society of Anesthesiologists, 93, 409-417. https://doi.org/10.1097/00000542-200008000-00019

[25] Exadaktylos, A.K., Buggy, D.J., Moriarty, D.C., Mascha, E. and Sessler, D.I. (2006) Can Anesthetic Technique for Primary Breast Cancer Surgery Affect Recurrence or Metastasis? The Journal of the American Society of Anesthesiologists, 105, 660-664. https://doi.org/10.1097/00000542-200610000-00008

[26] Biki, B., Mascha, E., Moriarty, D.C., Fitzpatrick, J.M., Sessler, D.I. and Buggy, D.J. (2008) Anesthetic Technique for Radical Prostatectomy Surgery Affects Cancer Recurrence: A Retrospective Analysis. Anesthesiology: The Journal of the American Society of Anesthesiologists, 109, 180-187. https://doi.org/10.1097/ALN.0b013e31817f5b73

[27] Woolf, C.J. and Wall, P.D. (1986) Relative Effectiveness of C Primary Afferent Fibers of Different Origins in Evoking a Prolonged Facilitation of the Flexor Reflex in the Rat. The Journal of Neuroscience: The Official Journal of the Society for Neuroscience, 6, 1433-1442. https://doi.org/10.1523/JNEUROSCI.06-05-01433.1986

[28] Dahl, J.B. and Moiniche, S. (2004) Pre-Emptive Analgesia. British Medical Bulletin, 71, 13-27. https://doi.org/10.1093/bmb/ldh030

[29] Woolf, C.J. (2021) Evidence for a Central Component of Post-Injury Pain Hypersensitivity. Nature, 306, 686-688. https://doi.org/10.1038/306686a0

[30] Costigan, M. and Woolf, C.J. (2000) Pain: Molecular Mechanisms. The Journal of Pain: Official Journal of the American Pain Society, 1, 35-44. https://doi.org/10.1054/jpai.2000.9818

[31] Latremoliere, A. and Woolf, C.J. (2009) Central Sensitization: A Generator of Pain Hypersensitivity by Central Neural Plasticity. The Journal of Pain, 10, 895-926. https://doi.org/10.1016/j.jpain.2009.06.012

[32] Katz, J. and Seltzer, Z. (2009) Transition from Acute to Chronic Postsurgical Pain: Risk Factors and Protective Factors. Expert Review of Neurotherapeutics, 9, 723-744. https://doi.org/10.1586/ern.09.20

[33] Vadivelu, N., Mitra, S., Schermer, E., Kodumudi, V., Kaye, A.D. and Urman, R.D. (2014) Preventive Analgesia for Postoperative Pain Control: A Broader Concept. Local and Regional Anesthesia, 7, 17-22. https://doi.org/10.2147/LRA.S62160 
[34] Hjermstad, et al. (2011) Studies Comparing Numerical Rating Scales, Verbal Rating Scales, and Visual Analogue Scales for Assessment of Pain Intensity in Adults: A Systematic Literature Review. Journal of Pain and Symptom Management, 41, 1073-1093. https://doi.org/10.1016/j.jpainsymman.2010.08.016

[35] Williamson, A. and Hoggart, B. (2005) Pain: A Review of Three Commonly Used Pain Rating Scales. Journal of Clinical Nursing, 14, 798-804. https://doi.org/10.1111/j.1365-2702.2005.01121.x

[36] Kain, Z.N., et al. (2000) Preoperative Anxiety and Postoperative Pain in Women Undergoing Hysterectomy. A Repeated-Measures Design. Journal of Psychosomatic Research, 49, 417-422. https://doi.org/10.1016/S0022-3999(00)00189-6

[37] Kulkarni, et al. (2017) Factors Associated with Acute Postoperative Pain Following Breast Reconstruction. JPRAS Open, 11, 1-13. https://doi.org/10.1016/j.jpra.2016.08.005

[38] Kalkman, et al. (2003) Preoperative Prediction of Severe Postoperative Pain. Pain, 105, 415-423. https://doi.org/10.1016/S0304-3959(03)00252-5

[39] Scott, L.E., Clum, G.A. and Peoples, J.B. (1983) Preoperative Predictors of Postoperative Pain. Pain, 15, 283-293. https://doi.org/10.1016/0304-3959(83)90063-5

[40] Sinatra, R.S., de Leon-Cassasola, O.A. and Viscusi, E.R. (2009) Acute Pain Management. Cambridge University Press, Cambridge.

[41] Sinatra, R.S., Jahr, J.S. and Watkins-Pitchford, J.M. (2010) The Essence of Analgesia and Analgesics. Cambridge University Press, Cambridge.

https://doi.org/10.1017/CBO9780511841378

[42] Ellison, N. (2002) Goodman \& Gilman's the Pharmacological Basis of Therapeutics. Anesthesia \& Analgesia, 94, 1377.

https://doi.org/10.1097/00000539-200205000-00085

[43] Walder, B., Schafer, M., Henzi, I. and Tramer, M.R. (2001) Efficacy and Safety of Patient-Controlled Opioid Analgesia for Acute Postoperative Pain. Acta Anaesthesiologica Scandinavica, 45, 795-804. https://doi.org/10.1034/j.1399-6576.2001.045007795.x

[44] Macintyre, P.E. (2001) Safety and Efficacy of Patient-Controlled Analgesia. British Journal of Anaesthesia, 87, 36-46. https://doi.org/10.1093/bja/87.1.36

[45] Carroll, I.R., Angst, M.S. and Clark, J.D. (2004) Management of Perioperative Pain in Patients Chronically Consuming Opioids. Regional Anesthesia and Pain Medicine, 29, 576-591. https://doi.org/10.1097/00115550-200411000-00011

[46] Lee, M., Silverman, S., Hansen, H. and Patel, V. (2011) A Comprehensive Review of Opioid-Induced Hyperalgesia. Pain Physician, 14, 145-161.

[47] O’Neal, J.B. (2013) The Utility of Intravenous Acetaminophen in the Perioperative Period. Frontiers in Public Health, 1, 25. https://doi.org/10.3389/fpubh.2013.00025

[48] Khalili, G., et al. (2013) Effect of Preemptive and Preventive Acetaminophen on Postoperative Pain Score: A Randomized, Double-Blind Trial of Patients Undergoing Lower Extremity Surgery. Journal of Clinical Anesthesia, 25, 188-192. https://doi.org/10.1016/j.jclinane.2012.09.004

[49] Macario, A. and Royal, M.A. (2011) A Literature Review of Randomized Clinical Trials of Intravenous Acetaminophen (Paracetamol) for Acute Postoperative Pain. Pain Practice, 11, 290-296. https://doi.org/10.1111/j.1533-2500.2010.00426.x

[50] Candiotti, et al. (2010) Safety of Multiple-Dose Intravenous Acetaminophen in Adult Inpatients. Pain Medicine, 11, 1841-1848.

https://doi.org/10.1111/j.1526-4637.2010.00991.x 
[51] Gilron, I., Orr, E., Tu, D., O’neill, J.P., Zamora, J.E. and Bell, A.C. (2005) A Placebo-Controlled Randomized Clinical Trial of Perioperative Administration of Gabapentin, Rofecoxib and Their Combination for Spontaneous and Movement-Evoked Pain after Abdominal Hysterectomy. Pain, 113, 191-200. https://doi.org/10.1016/j.pain.2004.10.008

[52] Souter, A.J., Fredman, B. and White, P.F. (1994) Controversies in the Perioperative Use of Nonsterodial Antiinflammatory Drugs. Anesthesia \& Analgesia, 79, 1178-1190. https://doi.org/10.1213/00000539-199412000-00025

[53] Smith, L.A., Carroll, D., Edwards, J.E., et al. (2000) Single-Dose Ketorolac and Pethidine in Acute Postoperative Pain: Systematic Review with Meta-Analysis. British Journal of Anaesthesia, 84, 48-58. https://doi.org/10.1093/oxfordjournals.bja.a013381

[54] Camu, F., Beecher, T., Recker, D.P. and Verburg, K.M. (2002) Valdecoxib, a COX-2-Specific Inhibitor, Is an Efficacious, Opioid-Sparing Analgesic in Patients Undergoing Hip Arthroplasty. American Journal of Therapeutics, 9, 43-51. https://doi.org/10.1097/00045391-200201000-00009

[55] Reuben, S.S. and Connelly, N.R. (2000) Postoperative Analgesic Effects of Celecoxib or Rofecoxib after Spinal Fusion Surgery. Anesthesia \& Analgesia, 91, 1221-1225. https://doi.org/10.1213/00000539-200011000-00032

[56] Chang, C.Y., Challa, C.K., Shah, J. and Jean, D.E. (2014) Gabapentin in Acute Postoperative Pain Management. BioMed Research International, 2014, Article ID: 631756. https://doi.org/10.1155/2014/631756

[57] Eckhardt, K., Ammon, S., Hofmann, U., Riebe, A., Gugeler, N. and Mikus, G. (2000) Gabapentin Enhances the Analgesic Effect of Morphine in Healthy Volunteers. Anesthesia \& Analgesia, 91, 185-191. https://doi.org/10.1097/00000539-200007000-00035

[58] Hurley, R.W., et al. (2002) Gabapentin and Pregabalin Can Interact Synergistically with Naproxen to Produce Antihyperalgesia. Anesthesiology, 97, 1263-1273. https://doi.org/10.1097/00000542-200211000-00033

[59] Ucak, A., Onan, B., Sen, H., Selcuk, I., Turan, A. and Yilmaz, A.T. (2011) The Effects of Gabapentin on Acute and Chronic Postoperative Pain after Coronary Artery Bypass Graft Surgery. Journal of Cardiothoracic and Vascular Anesthesia, 25, 824-829. https://doi.org/10.1053/j.jvca.2010.11.017

[60] Zhai, L., Song, Z. and Liu, K. (2016) The Effect of Gabapentin on Acute Postoperative Pain in Patients Undergoing Total Knee Arthroplasty. Medicine, 95, e3673. https://doi.org/10.1097/MD.0000000000003673

[61] Clarke, H., Page, G.M., McCartney, C.J., Huang, A., Stratford, P., et al. (2015) Pregabalin Reduces Postoperative Opioid Consumption and Pain for 1 Week after Hospital Discharge, But Does Not Affect Function at 6 Weeks or 3 Months after Total Hip Arthroplasty. British Journal of Anaesthesia, 115, 903-911. https://doi.org/10.1093/bja/aev363

[62] Ajori, L., Nazari, L., Mazloomfard, M.M. and Amiri, Z. (2012) Effects of Gabapentin on Postoperative Pain, Nausea and Vomiting after Abdominal Hysterectomy: A Double Blind Randomized Clinical Trial. Archives of Gynecology and Obstetrics, 285, 677-682. https://doi.org/10.1007/s00404-011-2023-6

[63] Chou, R., Gordon, D.B., de Leon-Casasola, O.A., et al. (2016) Management of Postoperative Pain: A Clinical Practice Guideline from the American Pain Society, the American Society of Regional Anesthesia and Pain Medicine, and the American Society of Anesthesiologists' Committee on Regional Anesthesia, Executive Com- 
mittee, and Administrative Council. The Journal of Pain, 17, 131-157.

[64] Chan, A.K.M., Cheung, C.W. and Chong, Y.K. (2010) Alpha-2 Agonists in Acute Pain Management. Expert Opinion on Pharmacotherapy, 11, 2849-2868. https://doi.org/10.1517/14656566.2010.511613

[65] Blaudszun, G., Lysakowski, C., Elia, N. and Tramèr, M.R. (2012) Effect of Perioperative Systemic $\alpha 2$ Agonists on Postoperative Morphine Consumption and Pain Intensity Systematic Review and Meta-Analysis of Randomized Controlled Trials. The Journal of the American Society of Anesthesiologists, 116, 1312-1322. https://doi.org/10.1097/ALN.0b013e31825681cb

[66] Kelika, P. and Arun, J.M. (2017) Evaluation of Clonidine as an Adjuvant to Brachial Plexus Block and Its Comparison with Tramadol. Journal of Anaesthesiology Clinical Pharmacology, 33, 197-202.

[67] Mohamed, A.A., Fares, K.M. and Mohamed, S.A. (2012) Efficacy of Intrathecally Administered Dexmedetomidine versus Dexmedetomidine with Fentanyl in Patients Undergoing Major Abdominal Cancer Surgery. Pain Physician, 15, 339-348.

[68] Yazıcıoğlu, D., Çaparlar, C., Akkaya, T., Mercan, U., Kulaçoğlu, H., Yazicioğlu, D. and Caparlar, C. (2016) Tizanidine for the Management of Acute Postoperative Pain after Inguinal Hernia Repair: A Placebo-Controlled Double-Blind Trial. European Journal of Anaesthesiology (Lippincott Williams \& Wilkins), 33, 215-222. https://doi.org/10.1097/EJA.0000000000000371

[69] Walker, S.M., Macintyre, P.E., Visser, E., et al. (2006) Acute Pain Management: Current Best Evidence Provides Guide for Improved Practice. Pain Medicine, 7, 3-5. https://doi.org/10.1111/j.1526-4637.2006.00081.x

[70] Bell, R.F., Dahl, J.B., Moore, R.A. and Kalso, E. (2006) Perioperative Ketamine for Acute Postoperative Pain. Cochrane Database of Systematic Reviews, No. 1, CD004603. https://doi.org/10.1002/14651858.CD004603.pub2

[71] Laskowski, K., Stirling, A., McKay, W.P. and Lim, H.J. (2011) A Systematic Review of Intravenous Ketamine for Postoperative Analgesia. Canadian Journal of Anesthesia, 58, 911-923. https://doi.org/10.1007/s12630-011-9560-0

[72] Pendi, A., Field, R., Farhan, S.D., Eichler, M. and Bederman, S.S. (2018) Perioperative Ketamine for Analgesia in Spine Surgery: A Meta-Analysis of Randomized Controlled Trials. Spine (Phila Pa 1976), 43, E299-E307. https://doi.org/10.1097/BRS.0000000000002318

[73] Dahmani, S., et al. (2011) Ketamine for Perioperative Pain Management in Children: A Meta-Analysis of Published Studies. Pediatric Anesthesia, 21, 636-652. https://doi.org/10.1111/j.1460-9592.2011.03566.x

[74] Loftus, R.W., et al. (2010) Intraoperative Ketamine Reduces Perioperative Opiate Consumption in Opiate-Dependent Patients with Chronic Back Pain Undergoing Back Surgery. Anesthesiology, 113, 639-646.

https://doi.org/10.1097/ALN.0b013e3181e90914

[75] Gorlin, A.W., et al. (2016) Intravenous Sub-Anesthetic Ketamine for Perioperative Analgesia. Journal of Anaesthesiology Clinical Pharmacology, 32, 160-167. https://doi.org/10.4103/0970-9185.182085

[76] Koppert, W., et al. (2004) Perioperative Intravenous Lidocaine Has Preventive Effects on Postoperative Pain and Morphine Consumption after Major Abdominal Surgery. Anesthesia \& Analgesia, 98, 1050-1055. https://doi.org/10.1213/01.ANE.0000104582.71710.EE

[77] McCarthy, G.C., Megalla, S.A. and Habib, A.S. (2010) Impact of Intravenous Lido- 
caine Infusion on Postoperative Analgesia and Recovery from Surgery: A Systematic Review of Randomized Controlled Trials. Drugs, 70, 1149-1163. https://doi.org/10.2165/10898560-000000000-00000

[78] Nowak, L., et al. (1984) Magnesium Gates Glutamate-Activated Channels in Mouse Central Neurones. Nature, 307, 462-465. https://doi.org/10.1038/307462a0

[79] Ozcan, P.E., et al. (2007) Role of Magnesium Sulfate in Postoperative Pain Management for Patients Undergoing Thoracotomy. Journal of Cardiothoracic and Vascular Anesthesia, 21, 827-831. https://doi.org/10.1053/j.jvca.2006.11.013

[80] Levaux, C., et al. (2003) Effect of Intra-Operative Magnesium Sulphate on Pain Relief and Patient Comfort after Major Lumbar Orthopaedic Surgery. Anaesthesia, 58, 131-135. https://doi.org/10.1046/j.1365-2044.2003.02999.x

[81] Lysakowski, C., Dumont, L., Czarnetzki, C. and Tramr, M.R. (2007) Magnesium as an Adjuvant to Postoperative Analgesia: A Systematic Review of Randomized Trials. Anesthesia \& Analgesia, 104, 1532-1539. https://doi.org/10.1213/01.ane.0000261250.59984.cd

[82] Albrecht, E., Kirkham, K.R., Liu, S.S., et al. (2013) Peri-Operative Intravenous Administration of Magnesium Sulphate and Postoperative Pain: A Meta-Analysis. Anaesthesia, 68, 79-90. https://doi.org/10.1111/j.1365-2044.2012.07335.x

[83] Helander, E.M., Webb, M.P., Bias, M., Whang, E.E., Kaye, A.D. and Urman, R.D. (2017) A Comparison of Multimodal Analgesic Approaches in Institutional Enhanced Recovery after Surgery Protocols for Colorectal Surgery: Pharmacological Agents. Journal of Laparoendoscopic \& Advanced Surgical Techniques, 27, 903-908. https://doi.org/10.1089/lap.2017.0338

[84] Deer, T., Krames, E.S., Hassenbusch, S., Burton, A., Caraway, D., Dupen, S. and Kim, P. (2008) Future Directions for Intrathecal Pain Management: A Review and Update from the Interdisciplinary Polyanalgesic Consensus Conference 2007. Neuromodulation: Technology at the Neural Interface, 11, 92-97. https://doi.org/10.1111/j.1525-1403.2008.00148.x

[85] Helander, E.M., Menard, B.L., Harmon, C.M., et al. (2017) Multimodal Analgesia, Current Concepts, and Acute Pain Considerations. Current Pain and Headache Reports, 21, 3. https://doi.org/10.1007/s11916-017-0607-y

[86] Essving, P., Axelsson, K., berg, E., Spnnar, H., Gupta, A. and Lundin, A. (2011) Local Infiltration Analgesia versus Intrathecal Morphine for Postoperative Pain Management after Total Knee Arthroplasty: A Randomized Controlled Trial. Anesthesia \& Analgesia, 113, 926-933. https://doi.org/10.1213/ANE.0b013e3182288deb

[87] Eskicioglu, C., Forbes, S.S., Aarts, M., Okrainec, A. and McLeod, R.S. (2009) Enhanced Recovery after Surgery (ERAS) Programs for Patients Having Colorectal Surgery: A Meta-Analysis of Randomized Trials. Journal of Gastrointestinal Surgery, 13, 2321-2329. https://doi.org/10.1007/s11605-009-0927-2

[88] Karmakar, M.K. (2001) Thoracic Paravertebral Block. Anesthesiology. The Journal of the American Society of Anesthesiologists, 95, 771-780.

https://doi.org/10.1097/00000542-200109000-00033

[89] Kairaluoma, P.M., Bachmann, M.S., Rosenberg, P.H. and Pere, P.J. (2006) Preincisional Paravertebral Block Reduces the Prevalence of Chronic Pain after Breast Surgery. Anesthesia \& Analgesia, 103, 703-708. https://doi.org/10.1213/01.ane.0000230603.92574.4e

[90] Paul, J.E., Arya, A., Hurlburt, L., Cheng, J., Thabane, L., Tidy, A. and Murthy, Y. (2010) Femoral Nerve Block Improves Analgesia Outcomes after Total Knee Arthroplasty: A Meta-Analysis of Randomized Controlled Trials. Anesthesiology: The 
Journal of the American Society of Anesthesiologists, 113, 1144-1162. https://doi.org/10.1097/ALN.0b013e3181f4b18

[91] Luo, Y. and Anderson, T.A. (2016) Phantom Limb Pain: A Review. International Anesthesiology Clinics, 54, 121-139. https://doi.org/10.1097/AIA.0000000000000095

[92] Li, A., Montaño, Z., Chen, V.J. and Gold, J.I. (2011) Virtual Reality and Pain Management: Current Trends and Future Directions. Pain Management, 1, 147-157. https://doi.org/10.2217/pmt.10.15

[93] Hoffman, H.G., et al. (2007) The Analgesic Effects of Opioids and Immersive Virtual Reality Distraction: Evidence from Subjective and Functional Brain Imaging Assessments. Anesthesia \& Analgesia, 105, 1776-1783. https://doi.org/10.1213/01.ane.0000270205.45146.db

[94] Freynet, A. and Falcoz, P.E. (2010) Is Transcutaneous Electrical Nerve Stimulation Effective in Relieving Postoperative Pain after Thoracotomy? Interactive Cardiovascular and Thoracic Surgery, 10, 283-288. https://doi.org/10.1510/icvts.2009.219576

[95] DeSantana, J.M., Sluka, K.A. and Lauretti, G.R. (2009) High and Low Frequency TENS Reduce Postoperative Pain Intensity after Laparoscopic Tubal Ligation: A Randomized Controlled Trial. The Clinical Journal of Pain, 25, 12-19. https://doi.org/10.1097/AJP.0b013e31817d1070

[96] Vickers, A. and Zollman, C. (1999) ABC of Complementary Medicine. Acupuncture. BMJ (Clinical Research Ed.), 319, 973-976. https://doi.org/10.1136/bmj.319.7215.973

[97] Sun, Y., Gan, T.J., Dubose, J.W. and Habib, A.S. (2008) Acupuncture and Related Techniques for Postoperative Pain: A Systematic Review of Randomized Controlled Trials. British Journal of Anaesthesia, 101, 151-160. https://doi.org/10.1093/bja/aen146

[98] Wu, M.S., Chen, K.H., et al. (2016) The Efficacy of Acupuncture in Post-Operative Pain Management: A Systematic Review and Meta-Analysis. PLoS ONE, 11, e0150367. https://doi.org/10.1371/journal.pone.0150367 